Review Article

\title{
MODIFICATION OF GUMS BY PERIODATE OXIDATION: A NATURAL CROSS-LINKER
}

\author{
SUMA OOMMEN SEN ${ }^{a^{*}}$, AMIT KUMAR NAYAK ${ }^{\mathrm{b}}$, PRITESH DEVBHUTIc, AMITAVA GHOSH ${ }^{\mathrm{d}}$
}

aGupta College of Technological Sciences, Ashram More, G. T. Road, Asansol 713301, West Bengal, India, bSeemanta Institute of Pharmaceutical Sciences, Mayurbhanj 757086, Odisha, India, cNSHM College of Pharmaceutical Technology, 124, B. L. Saha Road, Kolkata 700053, India, dBengal College of Pharmaceutical Sciences and Research, B. R. B. Sarani, Bidhannagar, Durgapur 713212, West Bengal, India

Email: suma_kpa@yahoo.co.in

Received: 29 Nov 2018 Revised and Accepted: 17 May 2019

\section{ABSTRACT}

Scientists throughout the world are in search of novel modified biopolymer to fabricate smart drug delivery systems based on hydrogel formulations using several cross-linkers like glutaraldehyde, glyoxal, epichlorhydrin, adipic acid dihydrazide, carbodiimide, genipin, etc. Agents that are fused into the polymeric structure like isocyanates, glutaraldehyde, polyepoxides, etc., and are extremely toxic in nature. In addition, these are susceptible to percolate out into the body on biodegradation of polymeric structure. As an alternative to these toxic cross-linking agents, the periodate-Schiff base staining technique is widely being used for cross-linking in biology and biochemistry. The mechanism of this cross-linking technique is based on the reaction in-between the Schiff reagent and the aldehydes produced via the periodate oxidation. During the past few decades, several researchers have already been studied on the natural gums and also, developed their dialdehyde derivatives via the periodate oxidation technique. These periodate oxidized gums are being used to cross-link gelatin, other proteins and chitosan to develop various smart systems for drug delivery, tissue engineering, wound dressing, edible films, etc. The current review presents a comprehensive discussion of the available reported literature on the periodate oxidation of various gums and their use as natural cross-linker.

Keywords: Dialdehyde gums, Periodate oxidation, Schiff reagent, Cross-linker

(C) 2019 The Authors. Published by Innovare Academic Sciences Pvt Ltd. This is an open-access article under the CC BY license (http://creativecommons.org/licenses/by/4.0/] DOI: http://dx.doi.org/10.22159/ijpps.2019v11i7.31058

\section{INTRODUCTION}

Scientists are always striving to deliver dosage forms in the human body and these dosage forms are being prepared using various biocompatible as well as biodegradable polymeric systems, so that, the safe and efficacious delivery of drugs to the patients can be provided [1-4]. With the advent of the concept of novel drug delivery approaches, researchers throughout the world are engaged in developing different types of novel drug delivery systems with newly fabricated synthetic and/or natural polymers $[5,6]$. These often utilize the use of hazardous and toxic chemicals. Recent years, novel or smart drug delivery systems are fabricated by modifying the biopolymers through the reactions of photopolymerization of their monomers [7], cross-linking using various metal cations [8-10], and using chemical cross-linkers like glutaraldehyde, glyoxal, epichlorhydrin, adipic acid dihydrazide, carbodiimide, genipin, etc [11-13] or via enzymatic cross-linking reactions [14]. Amongst these, cross-linking by means of metal cations is regularly reversible in the body and also, may produce various cytotoxic results [15]. Agents that are fused into the polymeric structure like isocyanates, glutaraldehyde, polyepoxides, etc., and are extremely toxic in nature. In addition, these are susceptible to percolate out into the body on biodegradation of polymeric structure [16]. These toxicity occurrences by these cross-linkers are one of the important obstructions in the utilization of biopolymers in the formula of various drug delivery dosage forms because their leakage into body liquids even at the small concentrations may be disastrous in some instances [17]. Currently, the researchers are engaged in developing of the safer cross-linking agents through the concept of green chemistry [18]. Since the past few years, the green chemistry is being used to develop the avant-garde drug delivery systems and methodologies that are highly effectual and associated with very lower/less toxicity [18-19]. Oxidation of gums by the use of periodate ions is a typical technique, extensively employed for the structural determinations (i.e., glycol cleavage) of almost all the complex carbohydrates [20]. An old and widely used technique in biology and biochemistry is the periodate-Schiff base staining technique for polysaccharidic gums. The oxidation of polysaccharides generally produces the usual structural features like
$(1 \rightarrow 2)$-linked, $\quad(\notin 3)$-linked, $(\notin 4)$-linked $(¥ 6)$-linked, and terminal (non-reducing) hexose residues (e. g., $\beta$-D-glucose). For the oxidation of vicinal diols of polysaccharide structure, it is necessary that the hydroxyl groups $(-\mathrm{OH})$ should be adjusted in an axialequatorial or equatorial-equatorial position, whereas the vicinal- $\mathrm{OH}$ groups are not reacted in a rigid axial-axial position since the intermediate complex are not formed [20]. Due to the non-toxicity as well as biodegradability, various oxidized gums (polysaccharide dialdehydes) are now being investigated as potential cross-linking agents for proteins and amino group-containing polymer in the preparation of hydrogels. The mechanism of this cross-linking technique is based on the reaction in-between the Schiff reagent and the aldehydes produced via the periodate oxidation $[20,21]$. Several research works have already been performed on the periodate oxidation of various gums [21-23]. In this context, we have aimed to write this structured review of the already published research works for the last $25 \mathrm{y}$ related to use of oxidized gums (polysaccharide dialdehydes) as natural cross-linker to prepare drug delivery systems and scaffold systems for tissue engineering applications.

\section{Mechanism of periodate oxidation}

The reaction of periodate oxidation of polysaccharides is generally occurred via the wide cleavage of the polysaccharide-chains, where the vicinal diols (i.e., 1, 2-diols) are actually cleaved using periodic acid $\left(\mathrm{HIO}_{4}\right)$ into 2 carbonyl $(\mathrm{C}=0)$ compounds [20]. This reaction (i.e., periodate oxidation) is selective for the modification of vicinal diols. Primarily, the periodate reaction of polysaccharides initiates by means of the configuration of the cyclic periodate ester [21]. The mechanism of periodate oxidation of polysaccharides is significant. Therefore, the interest is spotlighted on the cleavage step, accordingly. Before that, the cyclic ester of periodate is developed as the alcohol reacts [20,21]. The ester of periodate experiences some rearrangement of the electrons that causes the cleaving of $\mathrm{C}-\mathrm{C}$ bonding to form two carbonyl $(\mathrm{C}=0)$ compounds.

Periodate oxidation of polysaccharides also caused by depolymerization, for instance, periodate oxidations of chitosan and alginates [36-37]. The exposed, reducing ends both the polysaccharides consume further periodate, thus, leading to the over- 
oxidation. There are many mechanisms of depolymerization of polysaccharides. At the reasonable pHs and temperatures also lots of dialdehyde derivatives of polysaccharides are extremely vulnerable to the alkali-catalyzed $\beta$-elimination and after that, the degradation by the action of free radicals may also cause [20]. The periodate itself is unstable in nature. It changes over time to generate free radicals, in particular in the presence of light and as a result of this, oxidation reactions are generally performed also in the absence of oxygen and in the dark, utilizing the freshly formed periodate solution(s). In addition, the insignificant quantities of trace metals have to be isolated as these can catalyze the free radicals' formations.

The common principles of periodate oxidation of polysaccharides demonstrated by the b-D-Glcp residues are presented in fig. 1 .

(i)

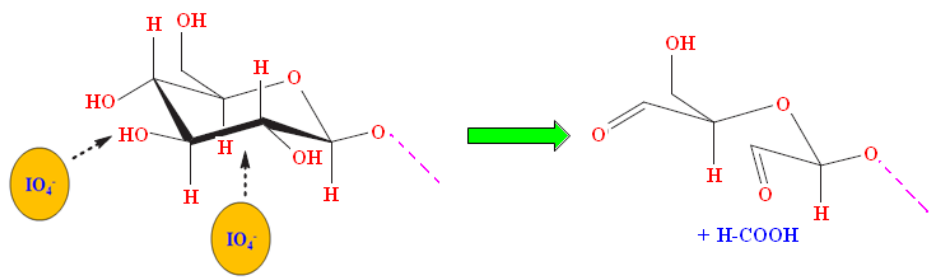

(ii)

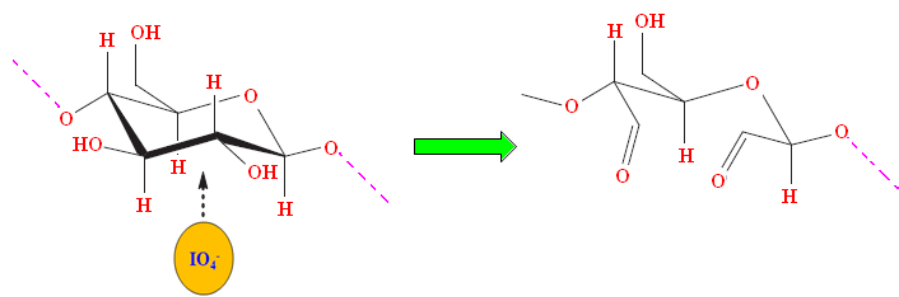

(iii)

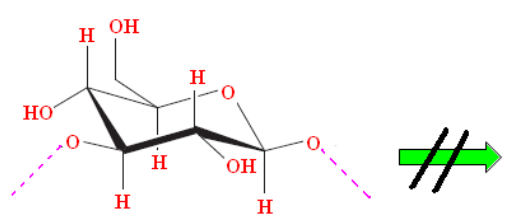

Fig. 1: General principles of periodate oxidation of polysaccharides demonstrated by the b-D-Glcp residues: (a) Terminal (non-reducing end) residues: double oxidation between $\mathrm{C} 2$ and $\mathrm{C} 3$ and between $\mathrm{C} 3$ and $\mathrm{C4}$, respectively, with the release of $\mathrm{C} 3$ as formaldehyde, (b) $(1 \rightarrow 4)$-linked residues where cleavage occurs between $\mathrm{C} 2$ and $\mathrm{C} 3$, (c) $(1 \rightarrow 3)$-linked residues (non-terminal), which are resistant to oxidation (Original drawing)

Malaprade [26] first observed the carbon-carbon (C-C) bonding cleavage in the compounds, where the carbon atoms (2 carbon atoms in the $\mathrm{C}-\mathrm{C}$ bonding) each having an oxygen atom either as a carbonyl $(\mathrm{C}=\mathrm{O})$ group or as a-OH group by periodates. The oxidation of alginate by periodate happens by the two different mechanisms: one mechanism is comparatively faster, which is not arbitrated by the hydroxyl free radicals; and another mechanism is comparatively slower because of lower concentrations of the hydroxyl radicals [27]. The cleavage of the occasional and atypical monomer in the polysaccharide structure of alginates causes the breakdown of the major polysaccharide chain of alginate molecules by the action of free radicals [27-29]. In the periodate oxidation of alginates, it has been observed that the periodate oxidation of alginates in the dilute solutions occurs at the initial stage in an unsystematic mode, that at a given time, one monomer-unit in a specified chain is only oxidized. Prior to the subsequent oxidative attack happens on the polysaccharidic chain, the protection of either one or both adjacentunit(s) occurs, instantaneously [28].

\section{Method of preparation of oxidized gums}

In the literature, the general methods of oxidation of a variety of gums are reported [30-31]. A specific concentration of gum solution is prepared and acidified to maintain $\mathrm{pH}$ at the lower range. Required concentrated sodium periodate or sodium metaperiodate solution is mixed with constant stirring at a specific temperature for a specific time period. The reaction vessel is kept in the dark to prevent auto-oxidation due to light. After a specific time interval the reaction process is stopped via the adding up of small amounts of ethylene glycol. Then oxidized gum is precipitated out by the addition of solvent.

In a study, Gupta et al., [22] prepared oxidized pectin using water and ethanol-water mixture. In the water medium, they added periodic acid ( $3 \mathrm{ml}$ of varying concentrations, 0.1 to $1.2 \mathrm{M}$ ) to a $2 \%$ of pectin solution in distilled water at the different $\mathrm{pH}$ milieu $(\mathrm{pH} 2$ 8) at the different ranges of temperatures (20 to $60^{\circ} \mathrm{C}$ ) for different time periods under dark condition. The oxidized pectin was precipitated out by employing an excess amount of isopropanol at the end of the reaction, and the oxidized pectin was vacuum filtered. They also carried out the oxidation of pectin using water-ethanol mixtures of varying ethanol contents (5 to $20 \%$ ). They observed around $20 \%$ alcoholic medium, lower $\mathrm{pH}$ and moderate temperature have increased aldehyde content. Dialdehyde tapioca starch was prepared using various concentrations of sodium metaperiodate at $\mathrm{pH} 3$ and $32^{\circ} \mathrm{C}$. The physicochemical properties of the dried product were investigated [32]. Li et al., [33] synthesized oxidized dextran and studied it's in situ crosslinking ability with phenylboronic modified chitosan.

\section{Oxidized gum synthesized via periodate oxidation reactions}

During the past few decades, the chemical modifications or functionalizations of different polysaccharidic gums are being investigated by various research groups $[3,34-36]$. The outcomes of these investigations present huge possibilities to transform these natural materials into various interesting as well as effective products for various biomedical applications including drug delivery, tissue engineering, wound healing, etc. [37]. In the occurrence of sodium periodate, the oxidation of gum is generally illustrated by the specific cleavage of the C2-C3 bond of residues, which results in the formation of aldehyde groups.

Gomez et al., [21] performed the periodate oxidation reactions on the- $\mathrm{OH}$ groups at $\mathrm{C} 2$ and $\mathrm{C} 3$ positions of the uronic units of sodium alginate structure by using sodium periodate. The effects of the degree of oxidation on physical characteristics of the oxidized derivatives (i.e., periodate-oxidized alginates) were evaluated. It was observed that the newer active functional groups in the alginate structure made the oxidized delivative of alginates as a very useful 
polymer, which can be used in the controlled drug delivery systems. In another study, functionalization of pectin via the periodate oxidation process was carried out and the study has also been suggested that periodate oxidized of pectin tenders the huge possibilities for the use in various biomedical applications like wound care applications [22]. In addition, the aldehyde groups introduced in the pectin molecular structure via the periodate oxidation reaction would help in in situ decrease of silver nitrate $\left(\mathrm{AgNO}_{3}\right)$ to produce nano-silver (metallic), which is also recognized as an outstanding anti-microbial material. Therefore, this approach can be successfully employed to design effectual wound dressings having biodegradability. The vicinal diols (i.e., 1, 2-diols) occurred at the carbons of $\mathrm{C} 2$ and $\mathrm{C} 3$ positions of anhydro d-glucopyranose ring in the molecular structure of pectin experience the periodate oxidation modification by using periodic acid to form a dialdehyde polysaccharidic structure of pectin (i.e., periodate-oxidized pectin) [22]. It has also been reported earlier that the formed aldehyde groups can react with 3-amino groups of hydroxylysine and lysine of gelatin to form the Schiff bases [38-39]. Kristiansen et al., [20] reviewed the periodate oxidation of polysaccharides for the modification of physical as well as chemical characteristics. The ring opening caused by the periodate oxidation is directed to the development of very much flexible 'hinges' in otherwise rather semiflexible or rigid structural features.

\section{Use of oxidized gum as cross-linked hydrogels}

In the earlier works by several groups of researchers, it is reported that the functionalization of polysaccharides by means of periodate oxidation because of the introduction of aldehyde functional groups within the carbohydrate molecular backbone [20]. These periodate oxidized polysaccharides can undergo reaction(s) with gelatin or other proteins $[23,37,40]$.

In the earlier reports, the researchers have observed that alginates (marine-derived natural biopolymer) are biocompatible and resistant to biodegradation [12]. However, periodate-oxidized alginates (dialdehyde derivative) are biodegradable in nature and used in diverse biomedical applications [21, 41]. Liu et al., [30] prepared oxidized sodium alginate hydrogel and investigated degradation performance. The degradation performance of hydrogel made of oxidized sodium alginate cross-linked with divalent calcium ions $\left(\mathrm{Ca}^{2+}\right)$ was characterized. The effect of oxidized sodium alginate hydrogels with various extents of oxidation was assessed as a function of degradation time by examining the changing of weight loss as well as molecular weight. The result of this study suggested that the degradation performance of the oxidized sodium alginate hydrogel found to depend on the extents of oxidation as well as the neighbouring medium. In addition, by altering the extent of oxidation, the degradation rates of oxidized sodium alginate hydrogels may be controlled. Yu et al., [42] prepared alginate dialdehyde as a naturally occurring cross-linker, and alginate dialdehyde was utilized for fixing the biological tissues. The results of the biological tissue-fixing indicated that the fixation index was almost similar to the glutaraldehyde-fixed counterparts and superior to the polyepoxy-fixed counterparts. In addition, the mechanical strength of alginate dialdehyde-fixed tissues was found comparable to the glutaraldehyde-fixed counterparts and superior to the polyepoxy-fixed counterparts. The results clearly demonstrated that the alginate dialdehyde is a cytocompatible cross-linker for the fixation of biological tissue.

In a study, Wan et al., [43] prepared oxidized alginate-based covalently cross-linked galactosylated chitosan scaffold. This biopolymeric scaffold was synthesized by the Schiff-base reaction devoid of using any toxic and corrosive chemical cross-linkers. They have shown that the in vitro equilibrium swelling and degradation rate of the scaffolds were found to be lessened with the increment of oxidized alginate content present in the scaffold formula. In addition, with the increment of oxidized alginate content, the thermal stability of the scaffolds was enhanced to some extent. These occurrences might be attributed to the difference of the extent of cross-linking and the characteristics of raw materials used to prepare these biopolymeric scaffolds. They also characterized these oxidized alginate-based biopolymeric scaffolds for the liver tissue engineering.
In other research, Matricardi et al., [44] characterized the degradation behaviour of alginates with the different degrees of oxidation and their capability to form polymeric gels in the occurrence of calcium $\left(\mathrm{Ca}^{2+}\right)$ ions. The interpenetrating polymer networks (IPNs) were prepared using the oxidized alginate (degrees of oxidation: 1 and $5 \%$ ) and hydroxyethyl-methacrylatederivatized dextran (dextran-HEMA). These IPN-based systems were evaluated for protein release. These IPN-based systems also demonstrated the characteristics, which were comparable to the IPNs made of native alginate, authenticating the suitability of these IPNs on the basis of oxidized alginate and dextran-HEMA as in situ forming hydrogels for the releasing of protein.

Several studies have recently been performed in the preparation of dialdehyde gums and their use as an effective cross-linker, whose aldehyde groups react with the 3-amino groups of hydroxylysine and lysine of protein molecules by the formations of Schiff's bases [38-39]. Jayakrishnan et al., [45] developed an in situ hydrogel made of periodate oxidized alginate and gelatine for wound dressing applications. Periodate-oxidized alginate is capable of speedy crosslinking of proteins, for example: gelatine, by the use of borax to form in situ periodate oxidized alginate-gelatine hydrogels. These hydrogels are biocomapatible as well as biodegradable in nature. In research by Balakrishnan et al., [38], an in situ hydrogel forming wound dressings made of gelatin and periodate-oxidized alginate were developed and investigated. The in situ hydrogel systems were prepared, which displayed super wound healing efficiency when these were evaluated on the full thickness of wounds in the rat models. Bigi et al., [46] investigated the chance of alginate dialdehyde in lower concentrations for the use of cross-linking and also, to stabilize the gelatine-based films. They also observed that the degree of cross-linking augmented as a function of alginate dialdehyde concentrations up to $23 \%$, approximately. Guo et al., [47] developed montmorillonite (an inorganic clay material) in carboxymethyl cellulose dialdehyde cross-linked gelatin-based films by applying the freezing-thawing method. In this research, the researchers observed that the prepared films possessed the better thermal stability with good mechanical characteristics. Therefore, these films can be suitably used as edible films.

Li et al., [33] developed a crosslinked hydrogel of periodate oxidized dextran and phenyl boronic modified chitosan to deliver the anticancer drug. They developed a novel kind of dually responsiveness (glucose and $\mathrm{pH}$ responsive) injectable hydrogel systems via the crosslinking of oxidized dextran and modified chitosan. The incorporation of cells, as well as drug molecules within these injectable hydrogel matrix systems, occurred due to the rapid gelation and biocompatible crosslinking by the in situ gel formation. Thus, in this work, they used the injectable hydrogels as a versatile material base for the anticancer drug delivery system. In another study, Murali et al., [31] synthesized poly (dialdehyde) guar gum via the oxidation of guar gum employing sodium periodate. Using the synthesized poly (dialdehyde) guar gum, they also prepared collagen-poly (dialdehyde) guar gum hybrid functionalized polymeric scaffolds for the use in tissue engineering. These 3 dimensional (3-D) porous scaffolds were immobilized covalently with the platelet-derived growth factor-BB. Guo et al., [23] synthesized oxidized xanthan gum with various aldehyde contents. In this work, xanthan gum was oxidized in the presence of sodium periodate to yield the corresponding C2/C3 dialdehyde product. Guo et al., [23] adjusted the aldehyde content of oxidized xanthan gum using different sodium periodate dosage that was quantified by titration. They also prepared the gelatin-oxidized xanthan gum based edible films by the casting method. In another study, Serrero et al., [48] investigated the cross-linking of oxidized starch and chitosan at different conditions to produce various bipolymeric systems. They studied their physical and mechanical properties. The overall results suggested that these bipolymeric systems made of cross-linked oxidized starch and chitosan can be used as biomaterials.

A schematic presentation of cross-linked hydrogel formation using oxidized gums (polysaccharide dialdehydes) and their applications is shown in fig. 2. The different polysaccharide dialdehydes studied as a cross-linker by several scientists is summarized and presented in table 1. 


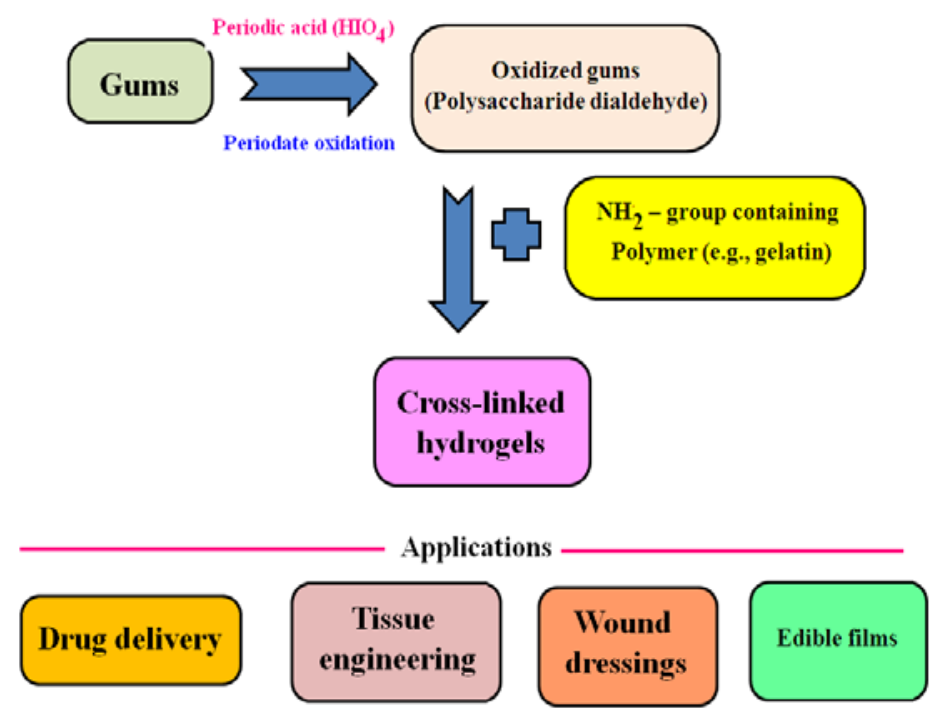

Fig. 2: Schematic presentation of cross-linked hydrogel formation using oxidized gums (polysaccharide dialdehydes) and their applications (original drawing)

Table 1: Preparation of polysaccharide dialdehyde and its use as crosslinker

\begin{tabular}{|c|c|c|c|c|}
\hline Polysaccharide used & $\begin{array}{l}\text { Reactant used to } \\
\text { form dialdehyde }\end{array}$ & Substance crosslinked & Applications & References \\
\hline Carboxymethyl cellulose & Sodium periodate & Gelatin & Edible films & [37] \\
\hline Xanthan gum & Sodium periodate & Gelatin & Edible films & [23] \\
\hline Dextran & Sodium periodate & Gelatin & Wound treatment & {$[40]$} \\
\hline Sodium alginate & Sodium per iodate & collagen & Tissue engineering & [49] \\
\hline Sodium alginate & Sodium per iodate & Biological tissues & Tissue engineering & {$[50]$} \\
\hline Gum Arabic & Sodium periodate & Gelatin & Spheroid cell culture & [51] \\
\hline Gum Arabic & Sodium periodate & Gelatin to prepare nanogel & Drug and gene delivery & [52] \\
\hline Dextran & Sodium periodate & Chitosan & Injectable tissue adhesive & [53] \\
\hline $\begin{array}{l}\text { Dextran, carboxymethyl } \\
\text { cellulose, sodium alginate, } \\
\text { and chondroitin }\end{array}$ & Sodium periodate & Amino glycoside antibiotics & $\begin{array}{l}\text { Smart hydrogels for topical } \\
\text { administration of amino glycoside } \\
\text { antibiotics }\end{array}$ & {$[54]$} \\
\hline Dextran & Sodium Periodate & $\begin{array}{l}\text { Thiol functionalized poly(amido- } \\
\text { amine) dendrimers }\end{array}$ & $\begin{array}{l}\text { Biocompatible injectable hydrogels and } \\
\text { potential tissue engineering material }\end{array}$ & {$[55]$} \\
\hline Starch & $\begin{array}{l}\text { Sodium meta } \\
\text { periodate }\end{array}$ & Chitosan & Biomedical application & {$[56]$} \\
\hline Gellan Gum & Sodium periodate & Hydrazide-modified hyaluronic acid & Biomedical application & [57] \\
\hline
\end{tabular}

\section{CONCLUSION}

The current review presents a comprehensive discussion of the available reported literature on the periodate oxidation of various polysaccharidic gums and their use as natural cross-linker. During the past few years, several oxidized gums are being synthesized with the help of periodate oxidation and these oxidized gums are also being employed as cross-linkers for amino acids or amino groupcontaining polymers. The cross-linking of oxidized gum and amino groups is predominantly because of the construction of imine bonds among aldehyde and amino moieties. In this review, we have discussed various important issues of periodate oxidations of gums such as mechanism of periodate oxidation, method of preparation of oxidized gums, oxidized gum as cross-linker and use of oxidized gum as a cross-linked hydrogel. The mechanism of this cross-linking technique is based on the reaction in-between the Schiff reagent and the aldehydes produced via the periodate oxidation. During the past few decades, several researchers have already been studied with the use of periodate oxidized gums to cross-link gelatin, other proteins or other gums/polymers to develop various smart systems for drug delivery tissue engineering applications, wound dressing applications, edible films, etc.

\section{ACKNOWLEDGMENT}

The first author thanks the management of Gupta College of Technological Sciences, Asansol, West Bengal, India for providing necessary supports to carry out this work.

\section{AUTHORS CONTRIBUTIONS}

All the authors have contributed equally

\section{CONFLICT OF INTERESTS}

There is no conflict of interest

\section{REFERENCES}

1. Patel DP, Singh S. Chitosan: a multifacet polymer. Int J Curr Pharm Res 2015;7:21-8.

2. Vijayanand P, Deepa A, Bhagavan Raju M. Development, characterization and evaluation of soft oral edible gel using gellan gum. Int J Appl Pharm 2017;9:73-7.

3. Ahmed VA, Goli D. Development and characterization of in situ gel of xanthan for ophthalmic formulation containing brimonidine tartrate. Asian J Pharm Clin Res 2018;11:277-84.

4. Toppo FA, Pawar RS. Novel drug delivery strategies and approaches for wound healing managements. J Crit Rev 2015;2:12-20.

5. Reddy PS, Bose PSC, Sruthi V, Saritha D. Investigation of kondagogu to develop transdermal film of repaglinide. Asian J Pharm Clin Res 2018;11:440-5.

6. Hasnain MS, Nayak AK. Chitosan as responsive polymer for drug delivery applications. In: Makhlouf ASH, Abu-Thabit NY. (eds.) Stimuli Responsive Polymeric Nanocarriers for Drug Delivery Applications. Vol. 1. Types and Triggers, Woodhead Publishing Series in Biomaterials, Elsevier Ltd; 2018. p. 581-605. 
7. Kim SH, Won CY, Chu CC. Synthesis and characterisation of dextran-maleic acid based hydrogel. J Biomed Mater Res 1999:46:160-70.

8. Nayak AK, Pal D. Plant-derived polymers: Ionically gelled sustained drug release systems. In: Mishra M. ed. Encyclopedia of Biomedical Polymers and Polymeric Biomaterials, Taylor and Francis Group, New York, NY 10017, U. S. A. Vol. VIII; 2016. p. 6002-17.

9. Pal D, Nayak AK. Alginates, blends and microspheres: controlled drug delivery. In: Mishra M. ed. Encyclopedia of Biomedical Polymers and Polymeric Biomaterials, Taylor and Francis Group, New York, NY 10017, U. S. A. Vol. I; 2015. p. 89-98.

10. Guru PR, Bera H, Das M, Hasnain MS, Nayak AK. Aceclofenacloaded Plantago ovata F. husk mucilage- $\mathrm{Zn}^{+2}$-pectinate controlled-release matrices. Starch-Starke 2018;70:1700136.

11. Jana S, Saha A, Nayak AK, Sen KK, Basu SK. Aceclofenac-loaded chitosan-tamarind seed polysaccharide interpenetrating polymeric network microparticles. Colloids Surf B: Biointerf 2013;105:303-9.

12. Bera H, Boddupalli S, Nayak AK. Mucoadhesive-floating zincpectinate-sterculia gum interpenetrating polymer network beads encapsulating ziprasidone $\mathrm{HCl}$. Carbohydr Polym 2015;131:108-18

13. Pal D, Nayak AK. Interpenetrating polymer networks (IPNs): Natural polymeric blends for drug delivery. In: Mishra M. ed. Encyclopedia of Biomedical Polymers and Polymeric Biomaterials, Taylor and Francis Group, New York, NY 10017, U. S. A. Vol. VI; 2015. p. 4120-30.

14. Chen T, Embree HD, Brown EM, Taylor MM, Payne GF. Enzymecatalyzed gel formation of gelatin and chitosan: potential for in situ applications. Biomater 2003;24:2831-41.

15. Lee KY, Mooney D. Hydrogels for tissue engineering. Chem Rev 2001;101:1869-79.

16. Kuijpers A, Engbers G, Krijgsveld J, Zaat SA, Dankert J, Feijen J. Crosslinking and characterization of gelatin matrices for biomedical applications. J Biomater Sci Polym 2000;11:225-43.

17. Speer D, Chavapil M, Eskelson C, Ulreich J. Biological effects of residual glutaraldehyde in glutaraldehyde-tanned collagen biomaterials. J Biomed Mater Res 1980;14:753-64.

18. Yogesh S, Ravikumar V, Anthony N, Douglas L. Applications of green chemistry in the manufacture of oligonucleotide drugs. Pure Appl Chem 2001:73:175-80.

19. Steven KM, John G, Chris D, Lisa N, David G, Alica W, et al. A green-by-design biocatalytic process for atorvastatin intermediate. Green Chem 2010;12:81-6.

20. Kristiansen KA, Potthast A, Christensen BE. Periodate oxidation of polysaccharides for modification of chemical and physical properties. Carbohydr Res 2010;345:1264-71.

21. Gomez CG, Rinaudo M, Villar MA. Oxidation of sodium alginate and characterization of the oxidized derivatives. Carbohydr Polym 2007;67:296-304.

22. Gupta B, Tummalapalli M, Deopura BL, Alam MS. Functionalization of pectin by periodate oxidation. Carbohydr Polym 2013;98:1160-5

23. Guo J, Ge L, Li X, Mu C, Li D. Periodate oxidation of xanthan gum and its crosslinking effects on gelatin-based edible films. Food Hydrocol 2014;39:243-50.

24. Perlin AS. Glycol-cleavage oxidation. Adv Carbohydr Chem Biochem 2006;60:183-250.

25. Larsen B, Painter T. The periodate-oxidation limit of alginate. J Carbohydr Res 1969;10:186-7.

26. Malaprade L. Action of polyalcohols on periodic acid. Analytical application. Bull Soc Chim 1928;43:683-96.

27. Scott JE, Tigwell MJ. On the mechanism of scission of alginate chains by periodate. Carbohydr Res 1976;47:105-17.

28. Painter T, Larsen B. Formation of hemiacetals between neighbouring hexuronic acid residues during periodate oxidation of alginate. Acta Chem Scand 1970;24:813-33.

29. Kang HA, Jeon GJ, Lee MY, Yang JW. Effectiveness test of alginate-derived polymeric surfactants. J Chem Technol Biotechnol 2002;77:205-10.

30. Liu M, Gao C, Chen J, Zhang X. Preparation and controlled degradation of oxidized sodium alginate hydrogel. Polym Degrad Stab 2009:94:1405-10.
31. Murali R, Thanikaivelan P, Cheirmadurai K. Collagenpoly(dialdehyde) guar gum based porous 3D scaffolds immobilized with growth factor for tissue engineering applications. Carbohydr Polym 2014;114:399-406.

32. Wongsagon R, Shobsngob S, Varavinit S. Preparation and physicochemical properties of dialdehyde tapioca starch. Starch/Starke 2005;57:166-72.

33. Li J, Hu W, Zhang Y, Tan H, Yan X, Zhao L, et al. $\mathrm{pH}$ and glucose dually responsive injectable hydrogel prepared by in situ crosslinking of phenylboronic modified chitosan and oxidized dextran. J Polym Sci Part A: Polym Chem 2015;53:1235-44.

34. Nayak AK, Bera H, Hasnain MS, Pal D. Graft-copolymerization of plant polysaccharides. In: VK Thakur. ed. Biopolymer Grafting, Synthesis and Properties, Elsevier Inc; 2018. p. 1-62.

35. Jana S, Maji N, Nayak AK, Sen KK, Basu SK. Development of chitosan-based nanoparticles through inter-polymeric complexation for oral drug delivery. Carbohydr Polym 2013;98:870-6.

36. Jana S, Das A, Nayak AK, Sen KK, Basu SK. Aceclofenac-loaded unsaturated esterified alginate/gellan gum microspheres: in vitro and in vivo assessment. Int J Biol Macromol 2013;57:129-37.

37. Mu C, Guo J, Li X, Lin W, Li DF. Preparation and properties of dialdehyde carboxymethyl cellulose crosslinked gelatin edible films. Food Hydrocol 2012;27:22-9.

38. Balakrishnan B, Jayakrishnan A. Self-cross-linking biopolymers as injectable in situ forming biodegradable scaffolds. Biomater 2005;26:3941-51.

39. Dash R, Foston M, Ragauskas A. Improving the mechanical and thermal properties of gelatin hydrogels cross-linked by cellulose nanowhiskers. Carbohydr Polym 2013:91;638-45.

40. Draye JP, Delaey, Voorde AV, Bulcke AV, Bogdanov B, Schacht E. In vitro release characteristics of bioactive molecules from dextran dialdehyde cross-linked gelatin hydrogel films. Biomater 1998;19:99-107.

41. Bouhadir K, Lee K, Alsberg E, Damm K, Anderson K, Mooney D. Degradation of partially oxidized alginate and its potential application for tissue engineering. Biotechnol Prog 2001;17:945-50.

42. Yu X, Xu Y, Li L, Gua Z, Zhang X. Feasibility study of a novel crosslinking reagent (alginate dialdehyde) for biological tissue fixation. Carbohydr Polym 2012;87:1589-95.

43. Wan C, Chen F, Tian M, Zhang D, Wang J, Wang Q, et al. Preparation and characterization of oxidized alginate covalently cross-linked galactosylated chitosan scaffold for liver tissue engineering. Mater Sci Eng C 2012;32:310-20.

44. Matricardi P, Pescosolidoa L, Piroa T, Vermondenb T, Covielloa $\mathrm{T}$, Alhaiquea F, Hennink WE. Biodegradable IPNs based on oxidized alginate and dextran-HEMA for controlled release of proteins. Carbohydr Polym 2011;86:208-13.

45. Jayakrishnan A, Balakrishnan B, Mohanty M, Umashankar PR. Evaluation of an in situ forming hydrogel wound dressing based on oxidized alginate and gelatine. Biomater 2005;26:6335-42.

46. Bigi A, Boanini E, Rubini K, Panzavolta S. Chemico-physical characterization of gelatin films modified with oxidized alginate. Acta Biomater 2010;6:383-8.

47. Guo J, Li X, Mu C, Zhang H, Qin P, Li D. Freezing-thawing effects on the properties of dialdehyde carboxymethyl cellulose crosslinked gelatin-MMT composite films. Food Hydrocol 2013;33:273-9.

48. Serrero A, Trombotto S, Cassagnau P, Bayon Y, Gravagna P, Montanari S, et al. Polysaccharide gels based on chitosan and modified starch: structural characterization and linear viscoelastic behavior. Biomacromol 2010;11:1534-43.

49. Hua Y, Liua L, Gu Z, Dana W, Dana N, Yu X. Modification of collagen with a natural derived cross-linker, alginate dialdehyde. Carbohydr Polym 2014;102:324-32.

50. Xu Y, Huang $\mathrm{C}, \mathrm{Li} \mathrm{L}, \mathrm{Yu} \mathrm{X}$, Wang $\mathrm{X}$, Peng $\mathrm{H}$, et al. In vitro enzymatic degradation of a biological tissue fixed by alginate dialdehyde. Carbohydr Polym 2013;95:148-54.

51. Sarika PR, Cinthya K, Jayakrishnan A, Anilkumar PR, James NR. Modified gum arabic cross-linked gelatin scaffold for biomedical applications. Mater Sci Eng C Mater Biol Appl 2014;43:272-9. 
52. Sarika PR, James NR. Preparation and characterisation of gelatin-gum arabic aldehyde nanogels via inverse miniemulsion technique. Int J Biol Macromol 2015;76:181-7.

53. Balakrishnan B, Soman D, Payanam U, Laurent A, Labarre D, Jayakrishnan A. A novel injectable tissue adhesive based on oxidized dextran and chitosan. Acta Biomater 2017;53:34354.

54. Hu J, Quan Y, Lai Y, Zheng Z, Hu Z, Wang X, et al. A smart aminoglycoside hydrogel with tunable gel degradation, ondemand drug release, and high antibacterial activity. J Controlled Release 2017;247:145-52.
55. Li S, Wang J, Song L, Zhou Y, Zhao J, Hou X, et al. Injectable PAMAM/ODex double-crosslinked hydrogels with high mechanical strength. Biomed Mater 2016;12:015012.

56. Serrero A, Trombotto S, Cassagnau P, Bayon Y, Gravagna P, Montanari S, et al. Polysaccharide gels based on chitosan and modified starch: structural characterization and linear viscoelastic behavior. Biomacromol 2010;11:1534-43.

57. Karvinen J, Koivisto JT, Jonkkari I, Kellomaki M. The production of injectable hydrazone crosslinked gellan gum-hyaluronan hydrogels with tunable mechanical and physical properties. Mech Behav Biomed Mater 2017;71:383-91. 